\title{
O impacto do volume informacional para aprendizes móveis
}

\author{
The impact of informational volume for mobile learners
}

Ana Valéria De Pieri Alvarez, Juliana Bueno

sobrecarga informacional, m-learning, design de informações

O crescimento do uso de tecnologias digitais, como smartphones, tem influenciado diretamente a forma como as pessoas vivem e interagem com o seu redor. Dentre as áreas afetadas está a educação, que passa a fazer o uso dessas tecnologias móveis, promovendo a mobilidade do estudante através do $\mathrm{m}$ learning (mobile-learning - aprendizado móvel). No entanto, esse contexto de mobilidade e suas consequências, muitas vezes não são considerados durante o planejamento, design e desenvolvimento de interfaces de m-learning, o que pode acarretar interações complexas, com informações excessivas ou de difícil compreensão, dificultando assim o processo de aprendizagem através da plataforma. Considerando esse contexto, o presente artigo visa entender como o volume informacional pode afetar o processo de aprendizagem do aprendiz móvel. Para isso, foram levantadas teorias acerca das temáticas de $m$-learning e sobrecarga informacional durante o aprendizado. Assim, foi possível observar que a característica de mobilidade do aprendiz móvel, o insere em um contexto com maior volume informacional, onde o mesmo recebe mais estímulos externos ao seu processo de aprendizagem. Essa sobrecarga informacional o distrai de sua tarefa atual de estudo, e interfere na atenção do mesmo, prejudicando a fixação do conteúdo estudado, e consequentemente seu processo de aprendizagem.

information overload, m-learning, information design

The usage growth of digital technologies, such as smartphones, has directly influenced the way people live and interact with their surroundings. Among the affected areas there is the education, which begins to use these mobile technologies, promoting student mobility through m-learning (mobile-learning). However, this mobility context and its consequences are not often considered during the planning, designing and development of m-learning interfaces, which can lead to complex interactions, with excessive or difficult information to understand, thus making the learning process through the platform harder. Considering this context, this article aims to understand how the informational volume can affect the learning process of the mobile learner. For that, theories about the themes of m-learning and information overload during learning were raised. Thus, it was possible to observe that the mobile learner's mobility characteristic insert them in a context with greater informational volume, where they receive more external stimuli to their learning process. This information overload distracts them from their current study task, and interferes with their attention, impairing the fixation of the studied content, and consequently their learning process.

\section{Introdução}

A popularização das tecnologias digitais e desenvolvimento de suas características de uso, impacta diretamente os comportamentos e hábitos humanos, modificando a forma como as interações sociais ocorrem (Bailer \& Tomitch, 2016; Padua e Jorente, 2018; Alexopoulou,

Anais do $10^{\circ} \mathrm{CIDI}$ e $10^{\circ} \mathrm{CONGIC}$

Kelli C.A.S. Smythe, Rafael de Castro Andrade (orgs.)

Sociedade Brasileira de Design da Informação - SBDI

Curitiba | Brasil | 2021
Proceedings of the $10^{\text {th }} \mathrm{CIDI}$ and $10^{\text {th }}$ CONGIC

Kelli C.A.S. Smythe, Rafael de Castro Andrade (orgs.)

Sociedade Brasileira de Design da Informação - SBDI Curitiba | Brazil | 2021 
Batsou \& Drigas, 2020). Dentre os aspectos que impactam esses comportamentos, podemos citar o aumento de possibilidades de uso dos dispositivos móveis, que é expandida principalmente pela sua conexão com a Internet (Criollo-C \& Luján-Mora, 2017).

O fácil acesso à Internet através de tecnologias móveis, como smartphones, permite que seus usuários tenham contato com um grande volume informacional, processo o qual Padua e Jorente (2018) consideram como a "revolução da informação". Essa onipresença tecnológica digital projeta ainda mais as mudanças nos padrões sociais, interferindo na forma como as pessoas trabalham, se socializam, se comunicam, se divertem, e aprendem (Criollo-C \& LujánMora, 2017; Alexopoulou, Batsou \& Drigas, 2020).

Considerando a educação como uma das áreas afetadas pelo aumento do uso de tecnologias móveis, podemos observar o crescimento do m-learning, que promove ao estudante a possibilidade de aprender em contexto de mobilidade. No entanto, esse contexto e suas consequências, muitas vezes não são considerados durante o planejamento e desenvolvimento de interfaces de m-learning, o que pode acarretar em interações complexas, com informações excessivas ou de difícil compreensão, dificultando assim o processo de aprendizagem através da plataforma.

O recorte do artigo advém de uma revisão de literatura sistemática (RBS) de um projeto de pesquisa maior, no qual foram buscadas relações sobre o uso de dispositivos móveis para a aprendizagem e questões cognitivas acerca do volume informacional gerado por essas tecnologias. Sobre o m-learning foi observado o crescimento do campo de pesquisa, considerando o aumento do uso de tecnologias digitais para diversos fins, que incluem o aprendizado (Bailer \& Tomitch, 2014). Como resultado da RBS, foi possível observar que a relação entre sobrecarga informacional e $m$-learning é academicamente pouco explorada, principalmente ao considerar a perspectiva de design das interfaces educacionais. Dentre os resultados desta pesquisa, Palalas $(2018,2019)$ destacou-se ao abordar o impacto da onipresença de dispositivos móveis ao aprendiz móvel, apresentando aspectos como a atenção dividida, a multitarefa, a dependência, e a sobrecarga cognitiva.

Essas características podem ser prejudiciais aos usuários de dispositivos móveis, principalmente considerando que ao utilizar essas tecnologias para o aprendizado, o estudante precisa focar sua atenção no conteúdo estudado, e não ser distraído por outros estímulos. Ao utilizar seus smartphones para aprendizagem, além de receber estímulos externos a essa tecnologia, como mudanças de iluminação ou ruído, os indivíduos também são bombardeados por um grande volume informacional proveniente do próprio dispositivo.

Palalas (2011) também defende que para um recurso de $m$-learning ser pedagogicamente sólido, é necessária uma investigação dos elementos visuais e informacionais nele utilizados. Com isso, podemos observar como o planejamento e design das informações educacionais é importante para o processo de aprendizagem dos aprendizes móveis. Pois, o volume informacional recebido pelo estudante durante sua interação com o dispositivo deve ser adequado para o processo de aprendizagem. Dessa forma, o design da informação deve atuar 
especificamente no controle informacional, propiciando ao usuário da interface uma interação equilibrada, que cumpra seus objetivos de compreensão e navegação.

Partindo desta contextualização, o presente artigo possui a problemática de: como o volume informacional pode afetar o processo de aprendizagem do aprendiz móvel? Assim, tomando como base literatura pertinente sobre $m$-learning e sobrecarga cognitiva durante a aprendizagem, o objetivo aqui é levantar as desvantagens da onipresença de dispositivos móveis para os aprendizes de m-learning, verificando assim, a relação do $m$-learning com a sobrecarga informacional. Entende-se que esta discussão inicial pode subsidiar, em pesquisas futuras, uma melhor verificação de como o design da informação pode auxiliar na proposta de novas soluções para o m-learning.

Por isso, neste artigo, inicialmente traz-se o referencial teórico sobre m-learning e o contexto do de mobilidade do aprendiz para melhor compreender o comportamento, e consequentes necessidades do estudante. Também a respeito de sobrecarga cognitiva, para verificar o impacto causado por muitos estímulos informacionais ao aprendiz móvel.

Em seguida, é apresentada uma discussão que relaciona a onipresença de dispositivos móveis com o aumento do volume informacional recebido pelo estudante móvel. Por fim, nas considerações finais são revisados os pontos de destaque apresentados no artigo.

\section{Referência teórico}

A fim de cumprir o objetivo proposto, foram levantadas teorias acerca de m-learning, como de Sharples et al. (2005, 2009, 2013, 2016), Coens, Reynvoet e Clarebout (2011) e Finardi, Leão e Amorim (2016), para melhor entender a área de estudo. Partindo das teorias de m-learning, buscou-se compreender o contexto de mobilidade do aprendiz, a fim de verificar seus comportamentos e suas necessidades durante o processo de aprendizagem móvel.

A respeito da temática de sobrecarga informacional, foram levantadas teorias como de Just e Buchweitz (2014) e Palalas $(2018,2019)$, a fim de melhor compreender o impacto do volume informacional durante o processo de aprendizagem.

\section{M-learning}

Ao abordar sobre o aprendizado, o acesso móvel à conteúdos educacionais, mediados por dispositivos como smartphones, promove um formato educacional conhecido como mobilelearning (aprendizagem móvel), popularmente conhecido por sua contração: m-learning. Apesar de não existir uma definição exata e estável de m-learning (Finardi, Leão \& Amorim, 2016; Pedro, Barbosa \& Santos, 2018), a distinção das duas abordagens apresentadas por Sharples (2013) é bem aceita nos estudos do campo. Estas são:

- aprendizagem assistida por tecnologia móvel;

- aprendizagem vinculada à mobilidade do aprendiz (não necessariamente à tecnologia). 
Ao focar na mobilidade do aprendiz, qualquer dispositivo que permita acesso flexível a conteúdos educacionais, independente de algum espaço físico específico, pode ser utilizado para esse aprendizado (Burston, 2014). Sharples, Taylor e Vavoula (2005, p. 5) exemplificam o uso de tecnologias como computadores, telefones móveis e fixos, e até mesmo livros ou blocos de notas para o aprendizado, defendendo que o "aluno que é móvel, e não a tecnologia". No entanto, na atual "era digital" dispositivos digitais comunicam um volume muito maior de estímulos e informações do que materiais como livros ou cadernos (Finardi, Leão \& Amorim, 2016).

Sharples et al. (2009) defendem que estamos em um período de mobilidade, tanto pessoal quanto técnica, onde dispositivos tecnológicos digitais de uso pessoal como telefones celulares, são transportados para todos os lugares. Esse acesso ubíquo promove a oportunidade de projetar novas formas de aprendizagem, ao conectar aprendizes de diferentes espaços, em diferentes contextos, conectando o mundo "real" e "virtual", e promovendo um ensino continuado (Sharples et al., 2009).

Para promover um bom processo de aprendizagem, que compreenda os comportamentos e necessidades dos aprendizes móveis, é necessário entender seus contextos e, para isso, é necessário entender como eles interagem com os materiais educacionais, e mapear as implicações da aprendizagem móvel (Sharples et al., 2009).

\section{Contexto do aprendiz móvel}

Dentre as implicações do $m$-learning, é importante destacar o contexto de mobilidade do estudante, considerando as diferentes experiências de aprendizagem que os alunos podem passam "em e entre" contextos (Sharples et al, 2009). Ao abordar o contexto do aprendiz, Sharples, Taylor e Vavoula (2016) defendem que este nunca é estático, já que o aprendizado não ocorre somente em um único contexto, mas que é resultado de uma interação contínua entre o estudante e seus recursos de aprendizagem.

Os autores também comentam que o uso de tecnologias móveis para a aprendizagem permite que seus estudantes aprendam enquanto exploram seus mundos, podendo se comunicar com outros alunos de diferentes contextos, que podem estar do outro lado do mundo, sendo parte de outras culturas, o que acrescenta ainda mais significado ao processo de aprendizagem (Sharples, Taylor \& Vavoula, 2016).

Ao abordar o contexto de mobilidade dos estudantes móveis, Kukulska-Hulme et al. (2011) o definem como peça central do processo de m-learning, e defendem que este é criado de forma contínua através da interação entre indivíduos (aprendizes e educadores), e das ferramentas que os cercam no dia a dia. Os autores também destacam cinco aspectos contextuais:

- mobilidade física, onde a localização se mostra relevante aos usuários, os quais buscam aprender durante os intervalos de suas atividades diárias;

- mobilidade tecnológica, que considera as tecnologias utilizadas para a aprendizagem; 
- mobilidade conceitual, onde o foco de atenção do estudante é considerado, já que este transita de seu objetivo conceitual de aprendizagem para outros, os quais são movidos por interesses pessoais, compromissos, ou curiosidades do indivíduo;

- mobilidade social, que considera que o aprendiz interage com diferentes grupos sociais, os quais contribuem de forma diferente para seu processo de aprendizagem;

- mobilidade temporal, que prevê que o aprendizado ocorre através de um processo acumulativo de diversos contatos e experiências com o conteúdo aprendido, sejam em formatos de aprendizagem formal ou informal.

A característica de mobilidade é frequentemente categorizada por pesquisadores em $\mathrm{m}$ learning como um aspecto positivo (Sharples et al., 2009; Kukulska-hulme et al., 2011). Além dela, dentre as vantagens do $m$-learning, é possível observar a aprendizagem situada e contextualizada (Campbell \& Geertsema, 2017; Palalas, 2018), a colaboração e engajamento entre estudantes (Campbell \& Geertsema 2017), a aprendizagem informal (Sharples, 2013), e a flexibilidade de uso (Sharples, 2013) como outros pontos positivos da modalidade.

Apesar do contexto de mobilidade oferecer aspectos positivos, também podemos observar pontos negativos a respeito do $m$-learning. Algumas desvantagens dizem respeito à questões tecnológicas, como capacidade limitada de armazenamento e processamento (Criollo-C \& Luján-Mora, 2017), ou problemas de usabilidade (Sharples, 2013).

Outras desvantagens da modalidade dizem respeito a forma como as pessoas utilizam as tecnologias durante o $m$-learning, tendo relação com questões pedagógicas, como treinamento limitado de profissionais da educação sobre o uso de novas tecnologias, ou mesma a falta de inovação e pouca a abertura para experimentar novas ferramentas de ensino (Criollo-C \& Luján-Mora, 2017). Também a "falta de desenvolvimento das habilidades cognitivas necessárias para a tarefa de aprendizagem e a incorporação incorreta das modalidade de interação disponíveis nos dispositivos m-learning para promover o processo de ensino" (Arce \& Pegueiros, 2017, p. 3).

Além de pontos negativos relacionados a questões tecnológicas e educacionais, também podemos observar desvantagens culturais, como a falta da apreciação do uso de dispositivos móveis como auxiliador do processo educacional, ou a resistências em ir além de práticas pedagógicas tradicionais (Criollo-C \& Luján-Mora, 2017).

Pontos que foram considerados positivos também podem possuir aspectos negativos à aprendizagem, como a característica de mobilidade. Coens, Reynvoet e Clarebout (2011) argumentam que os estudantes não estão mais limitados a aprender em um único ambiente, e que o uso de tecnologias móveis para a aprendizagem permite que os estudantes realizem outras atividades, ainda que em contexto educacional.

A aprendizagem torna-se independente do lugar. Isso significa que os alunos podem gastar seu tempo livre (por exemplo, enquanto esperam pelo ônibus, ou enquanto viajam de trem) de uma maneira significativa (...) Quando os alunos aprendem nesses momentos, na verdade estão realizando duas tarefas ao mesmo tempo: aprender enquanto esperam o ônibus ou viajam de trem. Além disso, a aprendizagem pode ser combinada com todos os tipos de atividades quando são usados dispositivos 
móveis, como dirigir um carro ou correr no parque. Os alunos têm a oportunidade de aprender enquanto realizam outra atividade e se tornam multitarefas educacionais (Coens, Reynvoet e Clarebout, 2011, p. 7).

Ao realizar o processo de aprendizagem em contexto de mobilidade, o estudante recebe um volume cada vez maior de estímulos externos, que podem contribuir ao seu aprendizado caso sejam condizentes ao conteúdo estudado, ou também podem prejudicar o estudo, ao se classificarem como informações distrativas. Esse grande volume informacional, o qual Just e Buchweitz (2014) se referem como "onipresença de fluxos de informações" tem crescido exponencialmente com o aumento do uso de tecnologias digitais como smartphones, tablets e computadores pessoais, sobrecarregando os estudantes com informações desnecessárias, e consequentemente distrativas à aprendizagem.

\section{Sobrecarga informacional}

A quantidade de informações que os usuários de dispositivos móveis têm acesso está crescendo exponencialmente por conta da popularização do uso dessas tecnologias. Esse volume informacional impacta diretamente a forma como esses indivíduos processam seus estímulos internos e externos, e interagem com seu redor (Just e Buchwitz, 2014; Bailer e Tomitch, 2016).

Considerando esse volume de dados produzido a partir do uso de tecnologias digitais, Palalas (2018) argumenta que essas informações são constantemente produzidas, sendo originadas de lugares como postagens em mídias sociais, sinais de GPS, registros de transações de compras, e registros de comunicação. A autora também comenta sobre como a quantidade de informações produzida tem aumentado com o passar dos anos.

Em 2010, o ex-CEO do Google, Eric Schmidt, apontou que criamos tanta informação em dois dias em 2010 quanto o fizemos desde o início do homem até 2003 (Siegler, 2010). Seis anos depois, em 2016, de acordo com IBM (2016), 2,5 quintilhões de bytes de dados são gerados todos os dias, com $90 \%$ dos dados no mundo hoje sendo criados apenas nos últimos dois anos. (...) Hilbert e López (2011) relataram que recebíamos cinco vezes mais informações todos os dias do que recebíamos em 1986. A capacidade de processar todas essas informações com computadores dobrou a cada 18 meses e com dispositivos de telecomunicações - a cada dois anos. (...) Desde a introdução da máquina de impressão de Gutenberg em meados do século 15, as pessoas têm reclamado da sobrecarga de informações. - superabundância de obras para leitura (...) Hoje em dia, devido à revolução digital, esse problema é exponencialmente maior. Portanto, a capacidade de destilar quais informações são válidas e valem a pena é mais crítica (Palalas, 2018. p. 5).

As informações que os aprendizes móveis recebem através de seus dispositivos móveis nem sempre são relevantes ao seu processo de aprendizagem. Palalas (2018) destaca que o constante fluxo de atualizações pode sobrecarregar e distrair os usuários, já que possibilita uma escolha infinita de recursos de informação, comunicação e entretenimento na palma da mão. A autora argumenta que a constante possibilidade de acesso móvel a materiais de aprendizagem pode levar o estudante à "hábitos de aprendizagem estressantes e 
improdutivos", caso estes alunos não sejam bem orientados e direcionados para a realização de um estudo estratégico e objetivo (Palalas, 2018, p. 4).

A grande quantidade de informações a que estamos expostos pode prejudicar nossa capacidade de selecionar e processar qualquer um desses recursos. Isso pode causar estresse e incapacidade de escolher informações válidas e tomar decisões (Palalas, 2018. p. 5).

Além do estresse e dificuldade em tomar decisões, Arce e Pegueiros (2017) defendem que o uso de dispositivos móveis durante a aprendizagem também impacta negativamente em habilidades cognitivas básicas como a memória. Os autores argumentam que isso ocorre porque o cérebro humano se adapta aos estímulos que recebe. Dessa forma, considerando que pode receber informações complexas de forma rápida e acessível através de tecnologias digitais, o cérebro passa a delegar a ação de lembrar informações, considerada uma habilidade cognitiva; e passa a somente a se preocupar em como buscar por essa informação, considerada uma habilidade procedimental (Arce \& Pegueiros, 2017). Esse comportamento pode ser considerado como negativo ao aprendizado, já que os indivíduos deixam de exercitar suas habilidades cognitivas, utilizando o acesso online à informações como muleta.

Ao receber esse grande volume informacional, podemos considerar que o contexto do estudante móvel é composto por diversos desafios como a distração e dificuldade de manter a atenção, a sobrecarga de informações, e o desejo de realizar mais de uma tarefa ao mesmo tempo. Palalas (2019) argumenta que, os estudantes móveis buscam realizar multitarefas a fim de atender ao grande número de responsabilidades que possuem. No entanto, a autora destaca que a realização concomitante dessas tarefas pode levar o estudante a estresse e frustração, já que o cérebro muda de uma tarefa para outra, não podendo focar completamente a atenção em nenhuma delas (Palalas, 2019).

Essa realização de duas tarefas ao mesmo tempo, e o contexto de multitarefa durante o processo educacional, exige demais do estudante. Just e Buchweitz (2014, p. 2) afirma que "a multitarefa eficaz requer que dois processos cognitivos complexos ocorram simultaneamente de forma harmoniosa enquanto compartilham alguma infraestrutura comum com recursos limitados". Ao realizar mais de uma tarefa ao mesmo tempo, a atividade cerebral envolvida não é resultado da soma do que seria a união das tarefas. Como os recursos cerebrais limitados, "não se pode coexecutar inúmeras tarefas ao mesmo tempo, sem afetar o desempenho" (Just \& Buchweitz, 2014, p. 7). Considerando o aprendizado nesse contexto de multitarefa educacional, não somente a execução das tarefas realizadas será comprometida, mas também a absorção do conteúdo estudado.

\section{Discussão}

Como podemos observar a partir do referencial teórico apresentado, o uso de tecnologias móveis permite que seus usuários tenham acesso a uma infinidade de informações na palma de suas mãos. Ainda que esses dispositivos facilitem os processos de comunicação digital, e 
potencializem o processo educacional através do $m$-learning, o volume informacional produzido através dessas tecnologias também pode gerar consequências negativas.

Dentre as desvantagens levantadas a respeito da onipresença de dispositivos móveis, além de aspectos tecnológicos relacionados à capacidade dos dispositivos e problemas de usabilidade (Criollo-C \& Luján-Mora, 2017), aspectos pedagógicos sobre a pouca abertura para o uso dessas tecnologias e sua incorporação incorreta (Criollo-C \& Luján-Mora, 2017; Pegueiros \& Pegueiros, 2017); e aspectos culturais sobre a falta de apreciação de dispositivos móveis e resistência ao seu uso, também foi observado que a característica de mobilidade também possui alguns pontos negativos, apesar de ser considerada, por muitos pesquisadores da área, como um ponto positivo de destaque.

O contexto de mobilidade pode impactar negativamente o estudante por conta do desvio de atenção que pode gerar. Ao interagir com diferentes ambientes, o estudante móvel recebe diversos estímulos externos que podem contribuir para o seu processo de aprendizagem, caso sejam relevantes para tal, ou podem distraí-lo, caso sejam informações distantes do conteúdo estudado.

O aumento exponencial das informações disponíveis aos usuários móveis os coloca em um estado de sobrecarga informacional, onde os mesmos precisam lidar com estímulos externos à tarefa por eles executada. Como Palalas (2019) explicita, esse maior volume informacional distrai os estudantes, gerando um processo de aprendizagem improdutivo e estressante. Este desvio de foco faz com que os mesmos precisem executar multitarefas concomitantemente, precisando interagir com sua tarefa atual de interação com o dispositivo móvel, enquanto dividem sua atenção com os demais estímulos à sua volta.

Como apontado por Just e Buchwietz (2014), ao realizar mais de uma tarefa ao mesmo tempo, o cérebro humano precisa dividir sua carga cognitiva entre as ações executadas, o que resulta em uma eficácia mais baixa, em pelo menos uma das tarefas realizadas. Ao levar em conta que a tarefa realizada no dispositivo móvel seja voltada à aprendizagem, esse desvio de atenção faz com que o aprendiz tenha seu processo de aprendizagem negativamente afetado, com menor fixação dos conteúdos estudados.

Para ilustrar a discussão apresentada, foi realizada uma síntese gráfica (vide Figura 1), que reúne seus pontos de desvantagens do uso de dispositivos móveis para aprendizagem, apresentando: questões tecnológicas, pedagógicas, culturais e cognitivas. 
Figura 1: Desvantagens do uso de dispositivos móveis para aprendizagem. Fonte: as autoras.
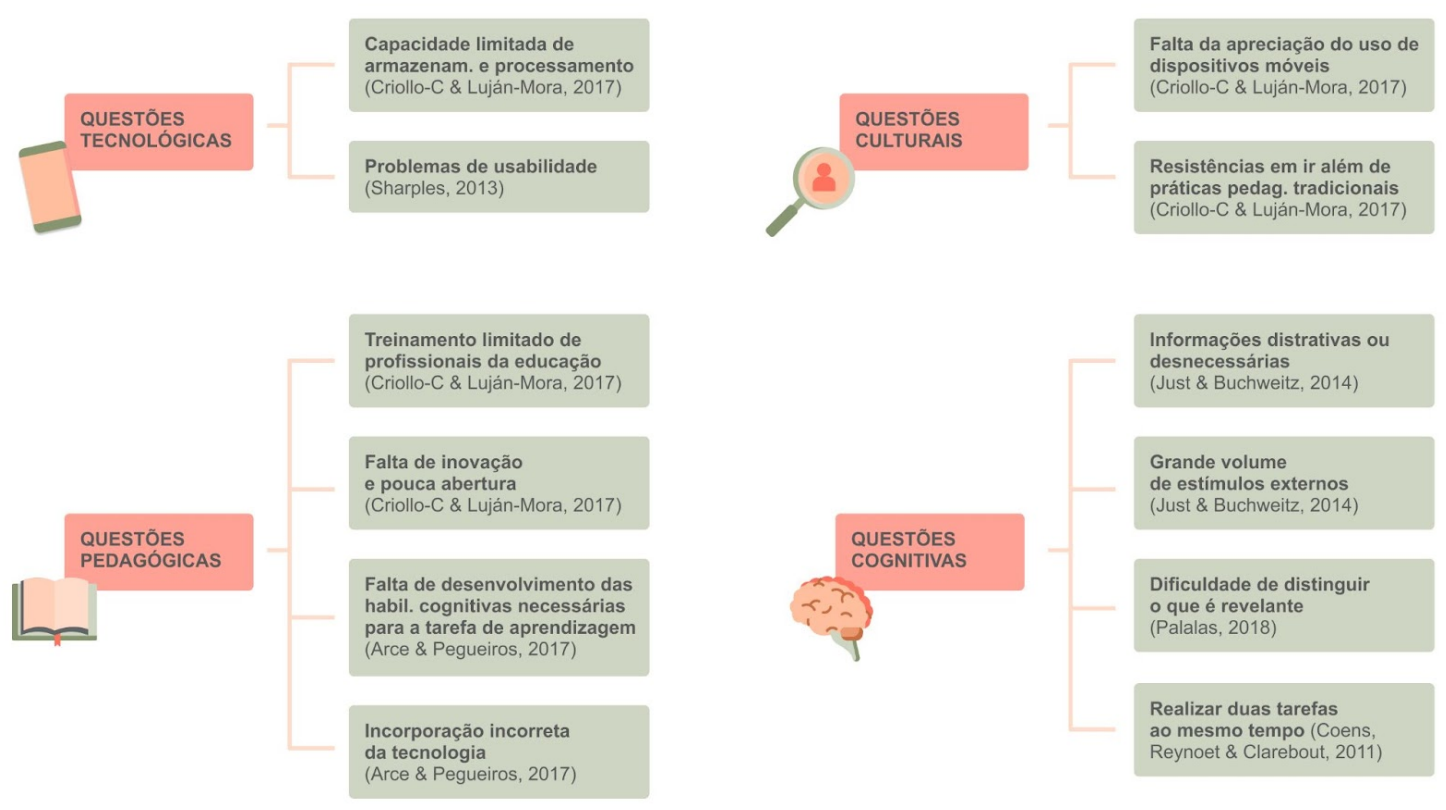

Considerando as desvantagens cognitivas apresentadas, o uso de dispositivos móveis para o aprendizado pode gerar uma possível sobrecarga cognitiva. Nesse aspecto o design da informação se mostra necessário para auxiliar na filtragem, organização e disposição das informações necessárias para o aprendizado. A aplicação de boas práticas de design na estrutura do layout, na escolha dos elementos visuais como cores, figuras e tipografias, e a arquitetura da informação, propiciam um ambiente mais bem estruturado, e mais propício para que o estudante possa focar em conteúdos relevantes ao seu aprendizado, e não se distraia com conteúdos acessórios.

\section{Considerações Finais}

A onipresença dos dispositivos móveis impacta diretamente em como as pessoas vivem e interagem, afetando áreas como a educação, através da inserção dessas tecnologias.

O m-learning exemplifica esse impacto, influenciando na forma como os indivíduos recebem e interagem com informações educacionais.

Para que os aprendizes tenham uma boa experiência de aprendizagem móvel é necessário que os mesmos tenham contato com recursos educacionais bem estruturados, e para isso é preciso que estes recursos supram as necessidades de seus usuários, segundo seus contextos de uso. O contexto do aprendiz móvel parte da característica de mobilidade, onde o indivíduo recebe a inferência de um grande volume informacional, resultado do meio pelo qual este está inserido.

As interações que o aprendiz faz com seu dispositivo móvel, e com seu ambiente externo podem gerar informações excessivas ou de difícil compreensão, que podem sobrecarregar o indivíduo, prejudicando assim, seu processo de aprendizagem. 
Buscando compreender como o volume informacional pode afetar o processo de aprendizagem do aprendiz móvel, o objetivo deste artigo é levantar as desvantagens da onipresença de dispositivos móveis para os aprendizes de $m$-learning, considerando que os mesmos utilizem dessas tecnologias como recurso educacional para o aprendizado móvel. A partir do referencial teórico e da discussão apresentados, foram observadas desvantagens acerca de questões tecnológicas, pedagógicas e culturais.

Dentre elas se destacaram os aspectos de atenção dividida, multitarefa durante a aprendizagem e sobrecarga informacional, os quais são impulsionados através do contexto de mobilidade do aprendiz. Considerando essas desvantagens, o design de informação pode atuar para diminuir o volume informacional acessório ou distrativo, a fim de apresentar ao aprendiz somente as informações necessárias ao processo de aprendizagem, o tornando, assim, mais eficaz.

\section{Referências}

Alexopoulou, A., Batsou, A., \& Drigas, A. (2020). Mobiles and Cognition: The Associations Between Mobile Technology and Cognitive Flexibility.

Arce, J. R., \& Pegueros, J. P. C. J. (2017). Impacto del m-learning en el proceso de aprendizaje: habilidades y conocimiento/The Impact Of m-learning On The Learning Process: Skills and Knowledge. RIDE Revista Iberoamericana para la Investigación y el Desarrollo Educativo, 8(15), 363-386.

Bailer, C., \& Tomitch, L. M. B. (2016). Estudos comportamentais e de neuroimagem sobre multitarefa: uma revisão de literatura. Alfa: Revista de Linguística (São José do Rio Preto), 60(2), 403-425.

Burston, J. (2013). Mobile-assisted language learning: A selected annotated bibliography of implementation studies 1994-2012. Language Learning \& Technology, 17(3), 157-225.

Campbell, C., \& Geertsema, M. (2017). Improving student language learning in adult education through the use of mobile learning: Barriers, challenges and ways to move forward. In Mobile learning in higher education in the Asia-Pacific region (pp. 541-553). Springer, Singapore.

Coens, J., Reynvoet, B., \& Clarebout, G. (2011). Mobile learning. Can students really multitask?. Journal of Educational Multimedia and Hypermedia, 20(1), 5-20.

Criollo-C, S., \& Luján-Mora, S. (2017, November). M-learning and their potential use in the higher education: a literature review. In 2017 International Conference on Information Systems and Computer Science (INCISCOS) (pp. 268-273). IEEE.

Finardi, K. R., Leao, R. G., \& Amorim, G. B. (2016). Mobile assisted language learning: Affordances and limitations of Duolingo. Education and Linguistics Research, 2(2), 48-65.

Just, M. A., \& Buchweitz, A. (2014). What brain imaging reveals about the nature of multitasking. The Oxford handbook of cognitive science, 1-16.

Kukulska-Hulme, A., Sharples, M., Milrad, M., Arnedillo-Sánchez, I., \& Vavoula, G. (2011). The genesis and development of mobile learning in Europe. In Combining e-learning and $\mathrm{m}$ learning: New applications of blended educational resources (pp. 151-177). IGI Global. 
Padua, M. C., \& Jorente, M. J. V. (2018). Design da Informação e Cognição: experiências confiáveis e significativas. In Congresso Internacional de Design de Informação (Vol. 8, No. 2017, pp. 1107-1120).

Palalas, A. (2011). Mobile-assisted language learning: Designing for your students. Second language teaching and learning with technology: views of emergent researchers, 71-94.

Palalas, A. (2018). Mindfulness in mobile and ubiquitous learning: Harnessing the power of attention. In Mobile and Ubiquitous Learning (pp. 19-44). Springer, Singapore.

Palalas, A. (2019). Mindfulness for human-centred digital learning. Argentinian Journal of Applied Linguistics.

Pedro, L. F. M. G., de Oliveira Barbosa, C. M. M., \& das Neves Santos, C. M. (2018). A critical review of mobile learning integration in formal educational contexts. International Journal of Educational Technology in Higher Education, 15(1), 1-15.

Sharples, M., Arnedillo-Sánchez, I., Milrad, M., \& Vavoula, G. (2009). Mobile learning. In Technology-enhanced learning (pp. 233-249). Springer, Dordrecht.

Sharples, M. (2013). Mobile learning: research, practice and challenges. Distance Education in China, 3(5), 5-11.

Sharples, M., Taylor, J., \& Vavoula, G. (2016). A Theory of learning for the mobile age. In The SAGEhandbook of e-learning research (pp. 63-81). SAGE.

\section{Sobre as autoras}

Ana Valéria De Pieri Alvarez, Mestranda do PPGDesign, UFPR, Brasil <ana.alvarez@ufpr.br> Juliana Bueno, Dra., UFPR, Brasil <julianabueno.ufpr@gmail.com> 